\title{
COVID-19 and antenatal care: An update
}

\author{
Mothusi Mncedisi Ncube ${ }^{1}$
}

\section{Dear Editor,}

COVID-19 infection, due to the severe acute respiratory syndrome coronavirus 2 (SARSCoV-2), has become a pandemic globally and has challenged many health systems with a shortage of resources and caused an outcry from all corners ${ }^{1}$. COVID-19 infection has shown detrimental effects on vulnerable people such as those with weak immune systems, among them the elderly and those with pre-existing illnesses ${ }^{2}$. With this in mind, questions arise on how this infection affects pregnant women and their unborn babies. The majority of pregnant women and newborns represent a unique population and are mostly healthy, but few may experience life events that bring physical, emotional, functional and social challenges and needs, requiring safe and personalised care during the current COVID-19 pandemic ${ }^{3}$. There are palpable fears of COVID-19 effects on pregnancy, although not much information is available to support this ${ }^{4}$. Over this time, the priority in pregnant women's care has been to reduce the transmission of COVID-19 and provide safe care to those susceptible to COVID-19.

At this point, research is still ongoing to determine the effects of COVID-19 infection on pregnancy and pregnant women in general ${ }^{5}$. However, the few data that exist do not show any detrimental effects on the growing baby or the mother herself, except for known respiratory signs and symptoms on affected patients ${ }^{6}$. The general presentation of this disease has been addressed extensively. At this point, many people know what signs and symptoms to look out for, such as fever, dry cough, shortness of breath or difficulty breathing, chills, fatigue, headache and sore throat ${ }^{7}$. Consequently, the general advice given to pregnant women is similar to that of the rest of the population. It includes social distancing, proper and frequent hand washing with soap and water, and use of sanitisers, and continued attendance of antenatal care advised by their healthcare providers ${ }^{8}$.

Attending antenatal care remains an integral part of maternity care as it allows for close monitoring of pregnancy development and management of disorders associated with the pregnancy and birth preparedness. The Royal College of Obstetricians and Gynaecologists (RCOG), the Royal College of Midwives and Ministry of Health and Wellness (MoHW) of Botswana suggest that pregnant women infected with COVID-19 are still most likely to experience no symptoms to mild illness from which they can make a full recovery. For those who develop severe symptoms or delayed recovery, it may suggest that a pregnant woman is developing a more significant chest infection that requires enhanced care ${ }^{9}$. The general advice to pregnant women like any other patient from the MoHW of Botswana is that whenever they experience COVID-19, they should contact an emergency number for further assistance. Evidence indicates that a flexible approach is critical to respond to fluctuations in risk from local or national COVID-19 prevalence and implications of regional or national public health policy ${ }^{3}$. With immunocompromised status coupled with physiological adaptive changes during pregnancy, it is reported that pregnant women could be more susceptible to COVID-19 infection than the general population ${ }^{10}$.

During this era of COVID-19, there is heightened alertness to the need for constant surveillance on everyone to keep them safe. Surely, COVID-19 will change the approach to the care of pregnant women, delivering mothers and post-delivery management. Birth plans and baby showers may have to be adjusted in consideration of COVID-19 prevention strategies. All pre-planned appointments must be honoured, and in cases of missed visits, communication of absence have to be made. With or without COVID-19, every pregnancy is important.

\section{AFFILIATION}

1 University of Botswana, School of Nursing, Gaborone, Botswana

\section{CORRESPONDENGE TO}

Mothusi Mncedisi Ncube.

University of Botswana School of Nursing, Private Bag UB 0022 ,

Block 230/217, Gaborone,

Botswana. E-mail: ncubem@ ub.ac.bw

\section{KEYWORDS}

COVID-19, antenatal care

Received: 20 January 2021

Accepted: 27 January 2021 


\section{REFERENGES}

1. Schwartz DA. An Analysis of 38 Pregnant Women With COVID-19, Their Newborn Infants, and Maternal-Fetal Transmission of SARS-CoV-2: Maternal Coronavirus Infections and Pregnancy Outcomes. Arch Pathol Lab Med. 2020;144(7):799-805. doi:10.5858/arpa.2020-0901-SA

2. Rasmussen SA, Smulian JC, Lednicky JA, Wen TS, Jamieson DJ. Coronavirus Disease 2019 (COVID-19) and pregnancy: what obstetricians need to know. Am J Obstet Gynecol. 2020;222(5):415-426. doi:10.1016/j.ajog.2020.02.017

3. Zaigham M, Andersson O. Maternal and perinatal outcomes with COVID-19: A systematic review of 108 pregnancies. Acta Obstet Gynecol Scand. 2020;99(7):823-829. doi:10.1111/aogs.13867

4. Lopes de Sousa ÁF, Carvalho HEF, Oliveira LB, et al. Effects of COVID-19 Infection during Pregnancy and Neonatal Prognosis: What Is the Evidence?. Int J Environ Res Public Health. 2020;17(11):4176. doi:10.3390/ijerph17114176

5. Koff WC, Williams MA. Covid-19 and Immunity in Aging Populations - A New Research Agenda. N Engl J Med. 2020;383(9):804-805. doi:10.1056/NEJMp2006761

6. Royal College of Obstetricians and Gynaecologists, The Royal College of Midwives. Guidance for provision of midwifeled settings and home birth in the evolving coronavirus (COVID-19) pandemic. https://www.rcog.org.uk/globalassets/ documents/guidelines/2020-07-10-guidance-for-provision-of-midwife-led.pdf. Published July 10, 2020. Accessed January 20, 2021.

7. Luo Y, Yin K. Management of pregnant women infected with COVID-19. Lancet Infect Dis. 2020;20(5):513-514. doi:10.1016/S1473-3099(20)30191-2

8. Walker PGT, Whittaker C, Watson O, et al. Report 12: The Global Impact of COVID-19 and Strategies for Mitigation and Suppression. London, United Kingdom: Imperial College London; 2020. doi:10.25561/77735

9. Begum MR, Ehsan N, Ehsan M, Sharif AB. Pregnancy and Childbirth in COVID-19 Positive/Probable and Suspected Patients: A Comprehensive Review. J Bangladesh Coll Phys Surg. 2020;38(COVID-19 Supplement):91-108. doi:10.3329/jbcps.v38iO.47443

10. Breslin N, Baptiste C, Gyamfi-Bannerman C, et al. Coronavirus disease 2019 infection among asymptomatic and symptomatic pregnant women: two weeks of confirmed presentations to an affiliated pair of New York City hospitals. Am J Obstet Gynecol MFM. 2020;2(2):100118. doi:10.1016/j.ajogmf.2020.100118 